\title{
On the renormalization of operator products: the scalar gluonic case
}

\author{
Max F. Zoller \\ Institut für Physik, University of Zurich (UZH), \\ Winterthurerstrasse 190, CH-8057 Zurich, Switzerland \\ E-mail: zoller@physik.uzh.ch
}

ABSTRACT: In this paper we study the renormalization of the product of two operators $O_{1}=-\frac{1}{4} G^{\mu \nu} G_{\mu \nu}$ in QCD. An insertion of two such operators $O_{1}(x) O_{1}(0)$ into a Greens function produces divergent contact terms for $x \rightarrow 0$.

In the course of the computation of the operator product expansion (OPE) of the correlator of two such operators $i \int \mathrm{d}^{4} x e^{i q x} T\left\{O_{1}(x) O_{1}(0)\right\}$ to three-loop order $[1,2]$ we discovered that divergent contact terms remain not only in the leading Wilson coefficient $C_{0}$, which is just the VEV of the correlator, but also in the Wilson coefficient $C_{1}$ in front of $O_{1}$. As this correlator plays an important role for example in QCD sum rules a full understanding of its renormalization is desireable.

This work explains how the divergences encountered in higher orders of an OPE of this correlator should be absorbed in counterterms and derives an additive renormalization constant for $C_{1}$ from first principles and to all orders in perturnbation theory. The method to derive the renormalization of this operator product is an extension of the ideas of [3] and can be generalized to other cases.

KeYwords: Perturbative QCD, Renormalization Group

ARXIV EPRINT: 1601.08094 


\section{Contents}

1 Introduction: the scalar gluonic operator $O_{1}$ and its correlator 1

2 Renormalization of $O_{1} \quad 3$

3 Renormalization of the product of two operators $O_{1} \quad 5$

4 Application to the OPE of the $O_{1} O_{1}$-correlator $\quad 7$

5 Conclusions $\quad 10$

\section{Introduction: the scalar gluonic operator $O_{1}$ and its correlator}

Local operators, i.e. products of fields at the same point in space-time, play an important role in quantum field theory (QFT) as they serve as building blocks for Lagrangians and Greens functions. The bilocal correlator of two such local operators is an important object in applications of QFT, such as sum rules. In this paper we study the renormalization of the scalar gluonic operator

$$
O_{1}(x):=-\frac{1}{4} G^{a \mu \nu} G_{\mu \nu}^{a}(x)
$$

constructed from the field strength tensor of QCD

$$
G_{\mu \nu}^{a}=\partial_{\mu} A_{\nu}^{a}-\partial_{\nu} A_{\mu}^{a}+g_{s} f^{a b c} A_{\mu}^{b} A_{\nu}^{c} .
$$

The operator (1.1) appears in the massless QCD Lagrangian

$$
\begin{aligned}
\mathcal{L}= & -\frac{1}{4} G_{\mu \nu}^{a} G^{a \mu \nu}-\frac{1}{2 \lambda}\left(\partial_{\mu} A^{a \mu}\right)^{2}+\partial_{\rho} \bar{c}^{a} \partial^{\rho} c^{a}+g_{s} f^{a b c} \partial_{\rho} \bar{c}^{a} A^{b} c^{c} \\
& +\bar{\psi}\left(\frac{i}{2} \overleftrightarrow{\not}-m\right) \psi+g_{s} \bar{\psi} A^{a} T^{a} \psi
\end{aligned}
$$

where $T^{a}$ are the generators and $f^{a b c}$ the structure constants of the gauge group.

A renormalized version of this operator, i.e. one which gives finite results if inserted into a Greens function, was obtained in [3, 4]. If we only consider matrix elements with physical external states and $m=0$ it can be renormalized multiplicatively:

$$
\left[O_{1}\right]=Z_{11} O_{1}^{\mathrm{B}}=-\frac{Z_{11}}{4} G^{\mathrm{B} a \mu \nu} G_{\mu \nu}^{\mathrm{B} a}
$$

where [...] marks the renormalized operator and the index B bare quantities, which means that all fields and couplings are replaced by bare ones. The renormalization constant derived in $[3,4]$

$$
Z_{11}=1+\alpha_{s} \frac{\partial}{\partial \alpha_{s}} \ln Z_{\alpha_{s}}=\left(1-\frac{\beta\left(\alpha_{s}\right)}{\varepsilon}\right)^{-1}
$$


can be expressed through the beta function

$$
\beta\left(\alpha_{s}\right)=\mu^{2} \frac{\mathrm{d}}{\mathrm{d} \mu^{2}} \ln \alpha_{s}=-\sum_{i \geq 0} \beta_{i}\left(\frac{\alpha_{s}}{\pi}\right)^{i+1}
$$

and at first order in $\alpha_{s}$ is equal to the renormalization constant for $\alpha_{s}: Z_{11}=Z_{\alpha_{s}}+\mathcal{O}\left(\alpha_{s}^{2}\right)$.

The bilocal correlator of this operator is defined as

$$
\widehat{\Pi}^{\mathrm{GG}}\left(q^{2}\right):=i \int \mathrm{d}^{4} x e^{i q x} T\left\{\left[O_{1}\right](x)\left[O_{1}\right](0)\right\}=i Z_{11}^{2} \int \mathrm{d}^{4} x e^{i q x} T\left\{O_{1}^{\mathrm{B}}(x) O_{1}^{\mathrm{B}}(0)\right\},
$$

The OPE of the correlator (1.7) (considering only scalar operators) reads

$$
\begin{aligned}
\widehat{\Pi}^{\mathrm{GG}}\left(q^{2}\right) & =q^{4} C_{0}^{\mathrm{GG}}\left(q^{2}\right) 1+C_{1}^{\mathrm{GG} \mathrm{B}}\left(q^{2}\right) O_{1}^{\mathrm{B}}+\sum_{i} C_{i}^{\mathrm{GG} \mathrm{B}}\left(q^{2}\right) O_{i}^{\mathrm{B}}+\mathcal{O}\left(\frac{1}{q^{2}}\right) \\
& =q^{4} C_{0}^{\mathrm{GG}}\left(q^{2}\right) 1+C_{1}^{\mathrm{GG}}\left(q^{2}\right)\left[O_{1}\right]+\sum_{i} C_{i}^{\mathrm{GG}}\left(q^{2}\right)\left[O_{i}\right]+\mathcal{O}\left(\frac{1}{q^{2}}\right),
\end{aligned}
$$

where the sum goes over a set of mass dimension four operators which form a suitable basis together with $O_{1}^{\mathrm{B}}$ or $\left[O_{1}\right]$ (see section 2). For sum rules (see e.g. [5]) we are usually interested in the vacuum expectation value (VEV) of the correlator

$$
q^{4} \Pi^{\mathrm{GG}}\left(q^{2}\right)=\left\langle 0\left|\widehat{\Pi}^{\mathrm{GG}}\left(q^{2}\right)\right| 0\right\rangle
$$

for large Euclidean momenta $-q^{2} \gg 0$. As the VEV of unphysical operators ${ }^{1}$ vanishes we can restrict ourselves to physical operators.

$C_{0}^{\mathrm{GG}}$ is known at four-loop level from [6] and $C_{1}^{\mathrm{GG}}$ at three-loop level from [2]. In [1,2] it was discovered, however, that the described renormalization procedure does not yield a finite result for $C_{1}^{\mathrm{GG}}$ starting from two-loop level. These divergent terms are proportional to $\delta^{(4)}(x)$ in $\mathrm{x}$-space and hence stem from the point where both operators $O_{1}$ in the correlator (1.7) are at the same point $x=0$. For this reason they are called contact terms. ${ }^{2}$

The complete renormalization of the operator product of two $O_{1}$ is also desirable for phenomenological applications in effective theories. An important example is double Higgs production in the framework of an effective theory with $m_{t} \rightarrow \infty$. Having integrated out the top loops the resulting vertices are $\propto O_{1} H$ and $\propto O_{1} H H$, where $H$ is the Higgs field (see e.g. [8]). the new renormalization constant $Z_{11}^{\mathrm{L}}$ defined below in (3.4) will be needed in a counterterm $\propto \mathrm{O}_{1} \mathrm{HH}$ if two effective vertices $\propto \mathrm{O}_{1} \mathrm{H}$ are inserted into two-loop diagrams with two external gluons, i.e. starting at one loop-order higher than the results presented in [8].

The paper is structured as follows: in section 2 the renormalization of Greens functions with one insertion of $O_{1}$ is reviewed following the ideas of [3]. This method is then extended in section 3 in order to renormalize the product of two such operators followed by the application of the found result to the OPE (1.8) in section 3 explaining the contact term in $C_{1}^{\mathrm{GG}}[1,2]$. We finish with some conclusions and acknowledgments.

\footnotetext{
${ }^{1}$ These are gauge dependent operators or operators which vanish due to equations of motion.

${ }^{2}$ In the case of the correlator of the pseudoscalar operator $\tilde{O}_{1}(x):=G^{a \mu \nu} G^{a \rho \sigma} \varepsilon_{\mu \nu \rho \sigma}$ it was proven in [7] that no contact terms can apear in $C_{1}$ which was explicitly confirmed in a three-loop calculation of this quantitiy in the same paper.
} 


\section{Renormalization of $O_{1}$}

For the purpose of this and the next section we rescale the field $A_{\mu}^{a} \rightarrow \frac{A_{\mu}^{a}}{g}$ transforming (1.3) into

$$
\begin{aligned}
& \mathcal{L}=-\frac{1}{4 g_{s}^{2}} G_{\mu \nu}^{a} G^{a \mu \nu}-\frac{1}{2 \lambda g_{s}^{2}}\left(\partial_{\mu} A^{a \mu}\right)^{2}+\partial_{\rho} \bar{c}^{a} \partial^{\rho} c^{a}+f^{a b c} \partial_{\rho} \bar{c}^{a} A^{b} c^{c} \\
& +\bar{\psi}\left(\frac{i}{2} \overleftrightarrow{\not b}-m\right) \psi+\bar{\psi} \mathscr{A}^{a} T^{a} \psi
\end{aligned}
$$

with the rescaled field strength tensor

$$
G_{\mu \nu}^{a}=\partial_{\mu} A_{\nu}^{a}-\partial_{\nu} A_{\mu}^{a}+f^{a b c} A_{\mu}^{b} A_{\nu}^{c} .
$$

We define the renormalization prescriptions

$$
A_{\mu}^{\mathrm{B} a}=A_{\mu}^{a} \frac{Z_{1}}{Z_{3}}, \quad c^{\mathrm{B} a}=c^{a} \sqrt{\tilde{Z}_{3}}, \quad \psi^{\mathrm{B}}=\psi \sqrt{Z_{2}}, \quad m^{\mathrm{B}}=m Z_{m}, \quad g_{s}^{\mathrm{B}}=g_{s} \frac{Z_{1}}{Z_{3}^{3 / 2}} \equiv g_{s} Z_{g},
$$

where $Z_{g}^{2}=Z_{\alpha_{s}}$ with $\alpha_{s}=\frac{g_{s}^{2}}{4 \pi}$. Hence

$$
\frac{A_{\mu}^{\mathrm{B} a}}{g_{s}^{\mathrm{B}}}=\frac{A_{\mu}^{a}}{g_{s}} \sqrt{Z_{3}}
$$

which is just the renormalization procedure for $A_{\mu}^{a}$ in the original Lagrangian (1.3). $Z_{1}$, $Z_{2}, Z_{3}, \tilde{Z}_{3}$ and $Z_{m}$ are therefore the usual renormalization constants of QCD. ${ }^{3}$ The bare Lagrangian reads

$$
\begin{aligned}
& \mathcal{L}_{\mathrm{B}}=-\frac{1}{4\left(g_{s}^{\mathrm{B}}\right)^{2}} G_{\mu \nu}^{\mathrm{B} a} G^{\mathrm{B} a \mu \nu}-\frac{1}{2 \lambda Z_{3}\left(g_{s}^{\mathrm{B}}\right)^{2}}\left(\partial_{\mu} A^{\mathrm{B} a \mu}\right)^{2}+\partial_{\rho} \bar{c}^{\mathrm{B} a} \partial^{\rho} c^{\mathrm{B} a} \\
& +f^{a b c} \partial_{\rho} \bar{c}^{\mathrm{B} a} A^{\mathrm{B} b} c^{\mathrm{B} c}+\bar{\psi}^{\mathrm{B}}\left(\frac{i}{2} \overleftrightarrow{\not \partial}-m_{\mathrm{B}}\right) \psi^{\mathrm{B}}+\bar{\psi}^{\mathrm{B}} A^{a \mathrm{~B}} T^{a} \psi^{\mathrm{B}}
\end{aligned}
$$

Finite results for Greens functions are usually obtained by applying the R-Operation (see e. g. $[9,10])$ to the unrenormalized Greens function or equivalently by using the bare Lagrangian in which a counterterm for every operator in the Lagrangian is defined. Finite Greens functions are derived from the generating functional of the path integral formalism

$$
\begin{aligned}
Z^{\mathrm{R}} & =\mathbf{R} \int \mathrm{d} \Phi \mathrm{e}^{i \int \mathrm{d}^{4} x(\mathcal{L}+J \cdot \Phi)} \\
& =\int \mathrm{d} \Phi \mathrm{e}^{i \int \mathrm{d}^{4} x\left(\mathcal{L}_{\mathrm{B}}+J \cdot \Phi\right)}
\end{aligned}
$$

with the multiplets of all fields in the Lagrangian and the respective external currents

$$
\Phi:=\left(\frac{A^{\mu}}{g_{s}}, \bar{c}, c, \bar{\psi}, \psi\right), \quad J:=\left(J_{\mu}, J_{\bar{c}}, J_{c}, J_{\bar{\psi}}, J_{\psi}\right)
$$

\footnotetext{
${ }^{3}$ The same as with unrescaled $A_{\mu}^{a}$ and the definition $A_{\mu}^{\mathrm{B} a}=A_{\mu}^{a} \sqrt{Z_{3}}$.
} 
and the integration measure

$$
\mathrm{d} \Phi:=\mathrm{d} A^{\mu} \mathrm{d} \bar{c} \mathrm{~d} c \mathrm{~d} \bar{\psi} \mathrm{d} \psi .
$$

A finite Greens function with the insertion of a local operator $\widetilde{O}_{i}(q)=\int \mathrm{d}^{4} x e^{i q x} O_{i}(x)$ is obtained as

$$
Z_{O_{i}}^{R}(q)=\mathbf{R} \int \mathrm{d} \Phi \widetilde{O}_{i}(q) e^{i \int \mathrm{d}^{4} x(\mathcal{L}+J \Phi)}
$$

which can also be written in terms of the bare Lagrangian and a superposition of bare local operators

$$
Z_{O_{i}}^{R}=Z_{i j} \int \mathrm{d} \Phi \widetilde{O}_{j}^{B}(q) e^{i \int \mathrm{d}^{4} x\left(\mathcal{L}_{B}+J \Phi\right)}
$$

In MS-like schemes the renormalization constants for these operators do not depend on $q$ and hence we set $q=0$. The important point now is that in renormalization schemes based on minimal subtraction the R-Operation commutes with the operation of taking derivatives w.r.t. the parameters of the theory $g_{s}, \lambda, \ldots$ and w.r.t. external currents. An operator insertion of $\widetilde{O}_{1} \equiv \widetilde{O}_{1}(0)$ in a Greens function can be obtained [3] by applying the operation

$$
\mathbf{D}_{1}:=\frac{1}{i}\left(\lambda \frac{\partial}{\partial \lambda}-\frac{1}{2} g_{s} \frac{\partial}{\partial g_{s}}-\frac{1}{2} J_{\mu} \frac{\delta}{\delta J_{\mu}}\right)
$$

to $(2.6)$ :

$$
\begin{aligned}
\mathbf{D}_{1} Z^{\mathrm{R}} & =\mathbf{D}_{1} \mathbf{R} \int \mathrm{d} \Phi \mathrm{e}^{i \int \mathrm{d}^{4} x(\mathcal{L}+J \cdot \Phi)}=\mathbf{R} \mathbf{D}_{1} \int \mathrm{d} \Phi \mathrm{e}^{i \int \mathrm{d}^{4} x(\mathcal{L}+J \cdot \Phi)} \\
& =\mathbf{R} \int \mathrm{d} \Phi \widetilde{O}_{1} \mathrm{e}^{i \int \mathrm{d}^{4} x(\mathcal{L}+J \cdot \Phi)}
\end{aligned}
$$

Using the representation (2.7) of $Z_{\mathrm{R}}$ we find that this equals

$$
\begin{aligned}
\mathbf{D}_{1} Z^{\mathrm{R}} & =\int \mathrm{d} \Phi\left\{\left(\lambda \frac{\partial}{\partial \lambda}-\frac{1}{2} g_{s} \frac{\partial}{\partial g_{s}}\right) \mathcal{L}_{\mathrm{B}}\right\} \mathrm{e}^{i \int \mathrm{d}^{4} x\left(\mathcal{L}_{\mathrm{B}}+J \cdot \Phi\right)} \\
& =\int \mathrm{d} \Phi\left\{\sum_{i=1}^{5} Z_{1 i} \widetilde{O}_{i}^{\mathrm{B}}\right\} \mathrm{e}^{i \int \mathrm{d}^{4} x\left(\mathcal{L}_{\mathrm{B}}+J \cdot \Phi\right)}
\end{aligned}
$$

A suitable basis of mass dimension four operators was given in [3]:

$$
\begin{aligned}
O_{1}= & -\frac{1}{4 g_{s}^{2}} G^{a \mu \nu} G_{\mu \nu}^{a}, \\
O_{2}= & m \bar{\psi} \psi, \\
O_{3}= & \bar{\psi}\left(\frac{i}{2} \overleftrightarrow{\not}-m\right) \psi, \\
O_{4}= & A_{\nu}^{a}\left\{\left(\delta^{a b} \partial_{\mu}-g f^{a b c} A_{\mu}^{c}\right) G^{b \mu \nu}+\bar{\psi} T^{a} \gamma^{\nu} \psi\right\} \\
& -\left(\partial_{\mu} \overline{c^{a}}\right)\left(\partial^{\mu} c^{a}\right), \\
O_{5}= & \left\{\left(\delta^{a b} \partial_{\mu}-f^{a b c} A_{\mu}^{c}\right) \partial^{\mu} \bar{c}^{a}\right\} c^{b} .
\end{aligned}
$$

Using (2.14) and collecting the coefficients in (2.15) we find the renormalization constants $Z_{1 i}$. The second line of (2.13) gives us the renormalized operator $\left[O_{1}\right]$, such that from $(2.13)$ 
and (2.15) we have $\left[O_{1}\right]=Z_{1 j} O_{j}^{\mathrm{B}}$. Similarly the renormalization constants for the other operators

$$
\left[O_{i}\right]=Z_{i j} O_{j}^{\mathrm{B}}
$$

are derived:

$$
Z_{i j}=\delta_{i j}+\bar{D}_{i} \ln \bar{Z}_{j} \quad(i, j \in\{1, \ldots, 5\})
$$

with

$$
\begin{aligned}
& \bar{D}_{1}=\lambda \frac{\partial}{\partial \lambda}-\alpha_{s} \frac{\partial}{\partial \alpha_{s}}, \quad \bar{D}_{2}=-m \frac{\partial}{\partial m}, \quad \bar{D}_{3}=0, \quad \bar{D}_{4}=2 \lambda \frac{\partial}{\partial \lambda}, \quad \bar{D}_{5}=0, \\
& \bar{Z}_{1}=Z_{\alpha}^{-1}, \quad \bar{Z}_{2}=Z_{m}^{-1}, \quad \bar{Z}_{3}=Z_{2}, \quad \bar{Z}_{4}=Z_{1} Z_{3}^{-1}, \quad \bar{Z}_{5}=Z_{3} Z_{1}^{-1} .
\end{aligned}
$$

These were first found in [3] and rederived for this study. ${ }^{4}$ The gauge-invariant operators (2.16) and (2.17) are physical operators of class $I$ according to the classification from $[3,11]$. In physical matrix elements the class $I$ operators do not vanish whereas the gauge-invariant class $I I^{a}$ operator (2.18) vanishes due to an equation of motion. The non gauge-invariant operators (2.19) and (2.20) are of class $I I^{b}$ and vanish due to a BRST identity in physical matrix elements. Hence in the massless case $O_{1}$ is renormalized multiplicatively with $Z_{11}$ as given in (1.5). In the following we set $m=0$.

\section{Renormalization of the product of two operators $O_{1}$}

We now want to apply this procedure in order to derive the renormalization constants for the insertion of two operators $\widetilde{O}_{1}$ into a Greens function. First (using (2.13)) we notice that

$$
\mathbf{D}_{1} \mathbf{D}_{1} Z^{\mathrm{R}}+i \mathbf{D}_{1} Z^{\mathrm{R}}=\mathbf{R} \int \mathrm{d} \Phi \widetilde{O}_{1} \widetilde{O}_{1} \mathrm{e}^{i \int \mathrm{d}^{4} x(\mathcal{L}+J \cdot \Phi)} .
$$

On the other hand

$$
\begin{aligned}
\left(\mathbf{D}_{1} \mathbf{D}_{1}+i \mathbf{D}_{1}\right) Z^{\mathrm{R}}= & \left(\mathbf{D}_{1} \mathbf{D}_{1}+i \mathbf{D}_{1}\right) \int \mathrm{d} \Phi \mathrm{e}^{i \int \mathrm{d}^{4} x\left(\mathcal{L}_{\mathrm{B}}+J \cdot \Phi\right)} \\
= & \left(\mathbf{D}_{1}+i\right) \int \mathrm{d} \Phi\left\{\sum_{i=1}^{5} Z_{1 i} \widetilde{O}_{i}^{\mathrm{B}}\right\} \mathrm{e}^{i \int \mathrm{d}^{4} x\left(\mathcal{L}_{\mathrm{B}}+J \cdot \Phi\right)} \\
= & \sum_{i, j=1}^{5} Z_{1 i} Z_{1 j} \int \mathrm{d} \Phi \widetilde{O}_{i}^{\mathrm{B}} \widetilde{O}_{j}^{\mathrm{B}} \mathrm{e}^{i \int \mathrm{d}^{4} x\left(\mathcal{L}_{\mathrm{B}}+J \cdot \Phi\right)} \\
& +\sum_{i=1}^{5}\left(\left(\mathbf{D}_{1} Z_{1 i}\right)+i Z_{1 i}\right) \int \mathrm{d} \Phi \widetilde{O}_{i}^{\mathrm{B}} \mathrm{e}^{i \int \mathrm{d}^{4} x\left(\mathcal{L}_{\mathrm{B}}+J \cdot \Phi\right)} \\
& +\sum_{i=1}^{5} Z_{1 i} \int \mathrm{d} \Phi\left(\mathbf{D}_{1} \widetilde{O}_{i}^{\mathrm{B}}\right) \mathrm{e}^{i \int \mathrm{d}^{4} x\left(\mathcal{L}_{\mathrm{B}}+J \cdot \Phi\right)} .
\end{aligned}
$$

\footnotetext{
${ }^{4}$ For the coefficients of the unphysical operators an additional "counting identity" is needed for which we refer to [3].
} 
This means that appart from the expected term $\sum_{i, j=1}^{5} Z_{1 i} Z_{1 j} O_{i}^{\mathrm{B}} O_{j}^{\mathrm{B}}=\left[O_{1}\right]\left[O_{1}\right]$ linear (L) terms of the form $i \sum_{i} Z_{1 i}^{\mathrm{L}} O_{i}^{\mathrm{B}}$ with new renormalization constants $Z_{1 i}^{\mathrm{L}}$ will in general contribute to the renormalization of an operator product:

$$
\left[O_{1}(x) O_{1}(0)\right]=\left[O_{1}(x)\right]\left[O_{1}(0)\right]+i \delta(x) \sum_{i} Z_{1 i}^{\mathrm{L}} O_{i}^{\mathrm{B}}(0) .
$$

A renormalized correlator should hence be defined as

$$
i \int \mathrm{d}^{4} x e^{i q x} T\left\{\left[O_{1}(x) O_{1}(0)\right]\right\}=i \int \mathrm{d}^{4} x e^{i q x} T\left\{\left[O_{1}(x)\right]\left[O_{1}(0)\right]\right\}-\sum_{i} Z_{1 i}^{\mathrm{L}} O_{i}^{\mathrm{B}} .
$$

We can again compute the first or second line of (3.2) and collect all fields and renormalization constants into local operators. In order to simplify the calculation we note that

$$
\int \mathrm{d}^{4} x \mathcal{L}_{\mathrm{B}}=\int \mathrm{d}^{4} x\left(O_{1}^{\mathrm{B}}(x)+O_{3}^{\mathrm{B}}(x)-O_{5}^{\mathrm{B}}(x)-\frac{1}{2 \lambda g_{s}^{2}}\left(\partial_{\mu} A^{a \mu}(x)\right)^{2}\right) .
$$

From (2.14) and (2.15) we find

$$
\mathbf{D}_{1}\left(\widetilde{O}_{1}^{\mathrm{B}}+\widetilde{O}_{3}^{\mathrm{B}}-\widetilde{O}_{5}^{\mathrm{B}}\right)=\sum_{i} Z_{1 i} \widetilde{O}_{i}
$$

We solve this for $\mathbf{D}_{1}\left(\widetilde{O}_{1}^{\mathrm{B}}\right)$ and plug it into the last three lines of (3.2). Then we discard all unphysical operators as well as their derivatives w.r.t. to $g_{s}$ and $\lambda$ as these will not contribute to physical matrix elements. ${ }^{5}$ This yields the result

$$
\begin{aligned}
Z_{11}^{\mathrm{L}} & =\frac{g_{s}^{2}}{2} \frac{Z_{g}^{\prime \prime}\left(g_{s}\right)}{Z_{g}}-\frac{3 g_{s}^{2}}{2}\left(\frac{Z_{g}^{\prime}\left(g_{s}\right)}{Z_{g}}\right)^{2}-\frac{g_{s}}{2} \frac{Z_{g}^{\prime}\left(g_{s}\right)}{Z_{g}} \\
& =-2 \alpha_{s}^{2}\left(\frac{Z_{\alpha_{s}}^{\prime}\left(\alpha_{s}\right)}{Z_{\alpha_{s}}}\right)^{2}+\alpha_{s}^{2} \frac{Z_{\alpha_{s}}^{\prime \prime}\left(\alpha_{s}\right)}{Z_{\alpha_{s}}} .
\end{aligned}
$$

We showed that the idea of [3] for the derivation of renormalization constants for dimension four operators can also be used for the derivation of renormalization constants of two such operator insertions. This method can be used for any operator as long as a combination of derivatives w.r.t. to external currents and parameters of the theory exists which produces an insertion of this operator into a Greens function starting from the generating functional $Z_{R}$ of the theory. In general, care has to be taken that contributions to different physical and unphysical operators are separated. Here we considered only one physical operator and discarded the unphysical ones.

Note that the procedure of inserting zero-momentum operators into $Z_{R}$ will produce only renormalization constants which are momentum independent and do not vanish for $q \rightarrow 0$. Hence we do not find a counterterm here which absorbs the contact terms in $C_{0}^{\mathrm{GG}}$

\footnotetext{
${ }^{5}$ For a full set of renormalization constants including the unphysical ones it is necessary to extend the set of unphysical operators as not all derivatives of $O_{3}^{\mathrm{B}}, O_{4}^{\mathrm{B}}, O_{5}^{\mathrm{B}}$ w.r.t. $g_{s}$ and $\lambda$ can be reabsorbed in exactly these operators.
} 
of (1.8). Such a counterterm $Z_{0}$ is $\propto q^{4}$ in momentum space. Accounting for this we can complete (3.4) and (3.3):

$$
\begin{aligned}
i \int \mathrm{d}^{4} x e^{i q x} T\left\{\left[O_{1}(x) O_{1}(0)\right]_{\text {full }}\right\} & =i \int \mathrm{d}^{4} x e^{i q x} T\left\{\left[O_{1}(x)\right]\left[O_{1}(0)\right]\right\}-\sum_{i} Z_{1 i}^{\mathrm{L}} O_{i}^{\mathrm{B}}-q^{4} Z_{0} \\
{\left[O_{1}(x) O_{1}(0)\right] } & =\left[O_{1}(x)\right]\left[O_{1}(0)\right]+i \delta(x) Z_{1 i}^{\mathrm{L}} O_{i}^{\mathrm{B}}(0)+i \square_{x}^{2} \delta(x) Z_{0}
\end{aligned}
$$

But deriving $Z_{0}$ from first principles is not within the reach this method.

\section{Application to the OPE of the $O_{1} O_{1}-$ correlator}

In $[1,2]$ the Wilson coefficient $C_{1}^{\mathrm{GG}}\left(q^{2}\right)$ was computed using the method of projectors $[12$, 13]. A projector $\mathbf{P}$ is applied to both sides of (1.8) which has the property $\mathbf{P}\left\{O_{1}^{\mathrm{B}}\right\}=1$ and $\mathbf{P}\left\{O_{i}^{\mathrm{B}}\right\}=0$ for $i \neq 1$. Thus we computed the bare Wilson coefficient via

$$
\mathbf{P}\left\{i \int \mathrm{d}^{4} x e^{i q x} T\left\{\left[O_{1}\right](x)\left[O_{1}\right](0)\right\}\right\}=\sum_{i} C_{i}^{\mathrm{B}}(q) \mathbf{P}\left\{O_{i}^{\mathrm{B}}\right\} \equiv C_{1}^{\mathrm{B}}(q) .
$$

with the projector $\mathbf{P}$ defined as: ${ }^{6}$

$$
C_{1}^{\mathrm{B}}(q)=\frac{\delta^{a b}}{n_{g}} \frac{g^{\mu_{1} \mu_{2}}}{(D-1)} \frac{1}{D} \frac{\partial}{\partial k_{1}} \cdot \frac{\partial}{\partial k_{2}}\left[\underset{\mu_{1}}{2}\right.
$$

where the blue circle represents the the sum of all bare Feynman diagrams which become 1PI after formal gluing (depicted as a dotted line in (4.2)) of the two external lines representing the operators on the l.h.s. of the OPE. These external legs carry the large Euclidean momentum q. If we use the fully renormalized current (3.4) we find

$$
\mathbf{P}\left\{i \int \mathrm{d}^{4} x e^{i q x} T\left\{\left[O_{1}(x) O_{1}\right](0)\right\}\right\}=\sum_{i} C_{i}^{\mathrm{B}}(q) \mathbf{P}\left\{O_{i}^{\mathrm{B}}\right\}-\sum_{i} Z_{1 i}^{\mathrm{L}} \mathbf{P}\left\{O_{i}^{\mathrm{B}}\right\} \equiv C_{1}^{\mathrm{B}}(q)-Z_{11}^{\mathrm{L}}
$$

and using (1.4) we find a fully renormalized Wilson coefficient as

$$
C_{1}^{\mathrm{ren}}=\frac{1}{Z_{11}} C_{1}^{\mathrm{B}}(q)-\frac{Z_{11}^{\mathrm{L}}}{Z_{11}}
$$

\footnotetext{
${ }^{6}$ The Feynman diagram was drawn with the Latex package Axodraw [14].
} 
From (1.5) and (3.7) we compute ${ }^{7}$

$$
\begin{aligned}
\frac{Z_{11}^{\mathrm{L}}}{Z_{11}}= & \frac{a_{s}^{2}}{\varepsilon}\left[-\frac{17 C_{A}^{2}}{24}+\frac{5 C_{A} n_{f} T_{F}}{12}+\frac{C_{F} n_{f} T_{F}}{4}\right] \\
+ & \frac{a_{s}^{3}}{\varepsilon}\left[\frac{1415 C_{A}^{2} n_{f} T_{F}}{864}-\frac{2857 C_{A}^{3}}{1728}+\frac{205 C_{A} C_{F} n_{f} T_{F}}{288}\right. \\
& \left.\quad-\frac{79 C_{A} n_{f}^{2} T_{F}^{2}}{432}-\frac{C_{F}^{2} n_{f} T_{F}}{16}-\frac{11 C_{F} n_{f}^{2} T_{F}^{2}}{72}\right] \\
+ & \frac{a_{s}^{3}}{\varepsilon^{2}}\left[-\frac{89 C_{A}^{2} n_{f} T_{F}}{144}+\frac{187 C_{A}^{3}}{288}-\frac{11 C_{A} C_{F} n_{f} T_{F}}{48}+\frac{5 C_{A} n_{f}^{2} T_{F}^{2}}{36}+\frac{C_{F} n_{f}^{2} T_{F}^{2}}{12}\right]
\end{aligned}
$$

which is exactly the contact term observed in $[1,2]$. Using

$$
\alpha_{s} \frac{Z_{\alpha_{s}}^{\prime}\left(\alpha_{s}\right)}{Z_{\alpha_{s}}}=-\frac{\beta\left(\alpha_{s}\right)}{\beta\left(\alpha_{s}\right)-\varepsilon}
$$

we arrive at

$$
\frac{Z_{11}^{\mathrm{L}}}{Z_{11}}=-a_{s}^{2} \frac{\beta_{1}}{\varepsilon}+a_{s}^{3}\left(\frac{\beta_{0} \beta_{1}}{\varepsilon^{2}}-\frac{2 \beta_{2}}{\varepsilon}\right)+a_{s}^{4}\left(-\frac{\beta_{0}^{2} \beta_{1}}{\varepsilon^{3}}+\frac{\beta_{1}^{2}+2 \beta_{0} \beta_{2}}{\varepsilon^{2}}-\frac{3 \beta_{3}}{\varepsilon}\right)+\mathcal{O}\left(\alpha_{s}^{5}\right)
$$

and as already suspected in [2] the contact term $\frac{Z_{11}^{\mathrm{L}}}{Z_{11}}$ or $Z_{11}^{\mathrm{L}}$ can indeed be expressed through the QCD $\beta$-function to all orders, namely

$$
\begin{aligned}
& Z_{11}^{\mathrm{L}}=\frac{1}{\varepsilon} \frac{-\beta\left(\alpha_{s}\right)+\alpha_{s} \beta^{\prime}\left(\alpha_{s}\right)}{\left(1-\frac{\beta\left(\alpha_{s}\right)}{\varepsilon}\right)^{2}}=\frac{1}{\varepsilon}\left(1-\frac{\beta\left(\alpha_{s}\right)}{\varepsilon}\right)^{-2} \alpha_{s}^{2} \frac{\partial}{\partial \alpha_{s}}\left[\frac{\beta\left(\alpha_{s}\right)}{\alpha_{s}}\right], \\
& \frac{Z_{11}^{\mathrm{L}}}{Z_{11}}=\frac{1}{\varepsilon} \frac{-\beta\left(\alpha_{s}\right)+\alpha_{s} \beta^{\prime}\left(\alpha_{s}\right)}{1-\frac{\beta\left(\alpha_{s}\right)}{\varepsilon}}=\frac{1}{\varepsilon}\left(1-\frac{\beta\left(\alpha_{s}\right)}{\varepsilon}\right)^{-1} \alpha_{s}^{2} \frac{\partial}{\partial \alpha_{s}}\left[\frac{\beta\left(\alpha_{s}\right)}{\alpha_{s}}\right] .
\end{aligned}
$$

Following the prescription of [17] we can derive the anomalous dimensions of the Wilson coefficients of the correlator (3.9) written as ${ }^{8}$

$$
\widehat{\Pi}_{\text {full }}^{\mathrm{GG}}:=i Z_{11}^{2} \int \mathrm{d}^{D} x e^{i q x} T\left\{O_{1}^{\mathrm{B}}(x) O_{1}^{\mathrm{B}}(0)\right\}-Z_{11}^{\mathrm{L}} O_{1}^{\mathrm{B}}-(\mu)^{-2} q^{4} Z_{0},
$$

We find

$$
\mu^{2} \frac{d}{d \mu^{2}} \widehat{\Pi}_{\text {full }}^{\mathrm{GG}}=2 \gamma_{11} \mu^{2} \frac{d}{d \mu^{2}} \widehat{\Pi}_{\text {full }}^{\mathrm{GG}}+\gamma_{11}^{L}\left[O_{1}\right]+\gamma_{0} q^{4}\left(\mu^{-2 \varepsilon}\right)
$$

\footnotetext{
${ }^{7}$ For this we need the QCD $\beta$-function at three-loop order $[15,16]$. All given results are in the $\overline{\mathrm{MS}}$-scheme. We define $a_{s}=\frac{\alpha_{s}}{\pi}=\frac{g_{s}^{2}}{4 \pi^{2}}$ and $l_{\mu q}=\ln \left(\frac{\mu^{2}}{-q^{2}}\right)$, where $\mu$ is the $\overline{\mathrm{MS}}$ renormalization scale. The number of active quark flavours is denoted by $n_{f}, C_{F}$ and $C_{A}$ are the quadratic Casimir operators of the quark and the adjoint representation of the gauge group and $n_{g}$ is the dimension of the adjoint representation. $T_{F}$ is defined through the relation $\operatorname{Tr}\left(T^{a} T^{b}\right)=T_{F} \delta^{a b}$.

${ }^{8}$ The renormalization scale $\mu$, which was omitted before for convenience, is carefully reintroduced. $D=$ $4-2 \varepsilon$ is the space-time dimension and $O_{1}^{\mathrm{B}}(x)$ has mass dimension $D$.
} 
and the anomalous dimensions are found to be

$$
\begin{aligned}
\gamma_{11} & =\mu^{2} \frac{d \log Z_{11}}{d \mu^{2}} \\
\gamma_{11}^{L} & =\left(-\mu^{2} \frac{d Z_{11}^{L}}{d \mu^{2}}+2 \gamma_{11} Z_{11}^{L}\right) \frac{1}{Z_{11}} \\
\gamma_{0} & =-\mu^{2} \frac{d Z_{0}}{d \mu^{2}}+\left(2 \gamma_{11}+\varepsilon\right) Z_{0}
\end{aligned}
$$

Applying these equations and the the well-known relation

$$
\mu^{2} \frac{d}{d \mu^{2}}=\alpha_{s}\left(\beta\left(\alpha_{s}\right)-\varepsilon\right) \frac{\partial}{\partial \alpha_{s}}+\mu^{2} \frac{\partial}{\partial \mu^{2}}
$$

to (4.8) and (1.5) we find (in the limit $\varepsilon \rightarrow 0$ ):

$$
\begin{aligned}
\gamma_{11} & =-\alpha_{s} \frac{\partial}{\partial \alpha_{s}} \beta\left(\alpha_{s}\right), \\
\gamma_{11}^{L} & =\alpha_{s}^{2} \frac{\partial^{2}}{\partial \alpha_{s}^{2}} \beta\left(\alpha_{s}\right),
\end{aligned}
$$

where (4.16) is in agreement with [3]. For $\gamma_{0}$ we cannot give a closed formula but a threeloop result. In the context of previous calculations [1,2] we computed the contact term of $C_{0}^{\mathrm{GG}}$, which equals $Z_{0}$, up to three-loop level: ${ }^{9}$

$$
\begin{aligned}
Z_{0}= & \frac{n_{g}}{16 \pi^{2}}\left\{\frac{1}{4 \varepsilon}+\frac{a_{s}}{\varepsilon}\left(\frac{17 C_{A}}{32}-\frac{5 n_{f} T_{F}}{24}\right)\right. \\
& +\frac{a_{s}^{2}}{\varepsilon}\left(\frac{11}{96} C_{A}^{2} \zeta_{3}+\frac{22351 C_{A}^{2}}{20736}-\frac{7}{24} C_{A} n_{f} T_{F} \zeta_{3}-\frac{799 C_{A} n_{f} T_{F}}{1296}\right. \\
& \left.\quad+\frac{1}{4} C_{F} n_{f} T_{F} \zeta_{3}-\frac{107}{288} C_{F} n_{f} T_{F}+\frac{49 n_{f}^{2} T_{F}^{2}}{1296}\right) \\
& +\frac{a_{s}}{\varepsilon^{2}}\left(\frac{n_{f} T_{F}}{12}-\frac{11 C_{A}}{48}\right) \\
& +\frac{a_{s}{ }^{2}}{\varepsilon^{2}}\left(-\frac{833 C_{A}^{2}}{1152}+\frac{73}{144} C_{A} n_{f} T_{F}+\frac{1}{12} C_{F} n_{f} T_{F}-\frac{5}{72} n_{f}^{2} T_{F}^{2}\right) \\
& \left.+\frac{a_{s}{ }^{2}}{\varepsilon^{3}}\left(\frac{121 C_{A}^{2}}{576}-\frac{11}{72} C_{A} n_{f} T_{F}+\frac{1}{36} n_{f}^{2} T_{F}^{2}\right)\right\},
\end{aligned}
$$

the full Wilson coefficient being

$$
\begin{aligned}
C_{0}^{\mathrm{GG}}= & Z_{0}+\frac{n_{g}}{16 \pi^{2}}\left\{\frac{l_{\mu q}}{4}+\frac{1}{4}\right. \\
+ & a_{s}\left(\frac{11}{48} C_{A} l_{\mu q}^{2}+\frac{73 C_{A} l_{\mu q}}{48}-\frac{3 C_{A} \zeta_{3}}{4}+\frac{485 C_{A}}{192}\right. \\
& \left.\quad-\frac{1}{12} l_{\mu q}^{2} n_{f} T_{F}-\frac{7}{12} l_{\mu q} n_{f} T_{F}-\frac{17 n_{f} T_{F}}{16}\right)
\end{aligned}
$$

\footnotetext{
${ }^{9}$ Only the Adler function of $C_{0}^{\mathrm{GG}}$ with the gauge group factors set to their QCD values was presented explicitly in [1].
} 


$$
\begin{aligned}
&+a_{s}^{2}\left(\frac{121}{576} C_{A}^{2} l_{\mu q}^{3}+\frac{313}{128} C_{A}^{2} l_{\mu q}^{2}-\frac{55}{32} C_{A}^{2} l_{\mu q} \zeta_{3}+\frac{37631 C_{A}^{2} l_{\mu q}}{3456}\right. \\
&-\frac{2059}{288} C_{A}^{2} \zeta_{3}+\frac{11}{64} C_{A}^{2} \zeta_{4}+\frac{25}{16} C_{A}^{2} \zeta 5+\frac{707201 C_{A}^{2}}{41472} \\
&-\frac{11}{72} C_{A} l_{\mu q}^{3} n_{f} T_{F}-\frac{85}{48} C_{A} l_{\mu q}^{2} n_{f} T_{F}-\frac{1}{8} C_{A} l_{\mu q} n_{f} T_{F} \zeta_{3} \\
&- \frac{6665}{864} C_{A} l_{\mu q} n_{f} T_{F}+\frac{169}{144} C_{A} n_{f} T_{F} \zeta_{3}-\frac{7}{16} C_{A} n_{f} T_{F} \zeta_{4} \\
&-\frac{7847}{648} C_{A} n_{f} T_{F}-\frac{1}{8} C_{F} l_{\mu q}^{2} n_{f} T_{F}+\frac{3}{4} C_{F} l_{\mu q} n_{f} T_{F} \zeta_{3} \\
&-\frac{131}{96} C_{F} l_{\mu q} n_{f} T_{F}+\frac{41}{24} C_{F} n_{f} T_{F} \zeta_{3}+\frac{3}{8} C_{F} n_{f} T_{F} \zeta_{4} \\
&-\frac{5281 C_{F} n_{f} T_{F}}{1728}+\frac{1}{36} l_{\mu q}^{3} n_{f}^{2} T_{F}^{2}+\frac{7}{24} l_{\mu q}^{2} n_{f}^{2} T_{F}^{2} \\
&\left.\left.+\frac{127}{108} l_{\mu q} n_{f}^{2} T_{F}^{2}+\frac{4715 n_{f}^{2} T_{F}^{2}}{2592}\right)\right\}
\end{aligned}
$$

which is known from $[18,19]$ and $[6]$ for the special case of the gauge group factors replaced by their QCD values. This leads to

$$
\begin{aligned}
\gamma_{0} & =\frac{n_{g}}{4}+\frac{1}{48} a_{s} n_{g}\left(51 C_{A}-20 n_{f} T_{F}\right) \\
& +\frac{a_{s}^{2} n_{g}}{6912}\left(C_{A}^{2}\left(2376 \zeta_{3}+22351\right)-16 C_{A} n_{f} T_{F}\left(378 \zeta_{3}+799\right)\right. \\
& \left.+8 n_{f} T_{F}\left(648 C_{F} \zeta_{3}-963 C_{F}+98 n_{f} T_{F}\right)\right) .
\end{aligned}
$$

\section{Conclusions}

I have presented a derivation of an additive counterterm $Z_{11}^{\mathrm{L}} O_{1}^{\mathrm{B}}$ needed to renormalize the correlator of two scalar gluonic operators $O_{1}$ using the path integral formalism and extending the ideas of [3]. This counterterm explains and absorbs the divergences found in the Wilson coefficient $C_{1}^{\mathrm{GG}}$ in $[1,2]$. A simple closed formula expressing $Z_{11}^{\mathrm{L}}$ to all orders through the QCD $\beta$-function was presented as well. Finally, the anomalous dimensions $\gamma_{11}$ and $\gamma_{11}^{L}$ for the correlator of two operators $O_{1}$ were expressed through the QCD $\beta$-function to all orders and the anomalous dimension $\gamma_{0}$ was computed at three-loop order.

\section{Acknowledgments}

I thank K. G. Chetyrkin for many useful discussions, for his comments on this paper and his collaboration on the previous project [1].

This research was supported in part by the Swiss National Science Foundation (SNF) under contract BSCGI0_157722.

Open Access. This article is distributed under the terms of the Creative Commons Attribution License (CC-BY 4.0), which permits any use, distribution and reproduction in any medium, provided the original author(s) and source are credited. 


\section{References}

[1] M.F. Zoller and K.G. Chetyrkin, OPE of the energy-momentum tensor correlator in massless QCD, JHEP 12 (2012) 119 [arXiv:1209.1516] [INSPIRE].

[2] M.F. Zoller, OPE of the energy-momentum tensor correlator and the gluon condensate operator in massless QCD to three-loop order, JHEP 10 (2014) 169 [arXiv:1407.6921] [INSPIRE].

[3] V. Spiridonov, Anomalous dimension of $g_{\mu \nu}^{2}$ and $\beta$-function, Preprint IYAI-P-0378 (1984).

[4] N.K. Nielsen, Gauge invariance and broken conformal symmetry, Nucl. Phys. B 97 (1975) 527 [inSPIRE].

[5] H. Forkel, Direct instantons, topological charge screening and QCD glueball sum rules, Phys. Rev. D 71 (2005) 054008 [hep-ph/0312049] [InSPIRE].

[6] P.A. Baikov and K.G. Chetyrkin, Top quark mediated Higgs boson decay into hadrons to order $\alpha_{s}^{5}$, Phys. Rev. Lett. 97 (2006) 061803 [hep-ph/0604194] [INSPIRE].

[7] M.F. Zoller, OPE of the pseudoscalar gluonium correlator in massless QCD to three-loop order, JHEP 07 (2013) 040 [arXiv: 1304.2232] [INSPIRE].

[8] J. Grigo, K. Melnikov and M. Steinhauser, Virtual corrections to Higgs boson pair production in the large top quark mass limit, Nucl. Phys. B 888 (2014) 17 [arXiv:1408.2422] [INSPIRE].

[9] W. E. Caswell and A.D. Kennedy, Simple approach to renormalization theory, Phys. Rev. D 25 (1982) 392.

[10] J. C. Collins, Renormalization : an introduction to renormalization, the renormalization group, and the operator-product expansion, Cambridge monographs on mathematical physics, Cambridge University Press, Cambridge U.K. (1984).

[11] W.S. Deans and J.A. Dixon, Theory of gauge invariant operators: their renormalization and S matrix elements, Phys. Rev. D 18 (1978) 1113 [INSPIRE].

[12] S.G. Gorishnii, S.A. Larin and F.V. Tkachov, The algorithm for OPE coefficient functions in the MS scheme, Phys. Lett. B 124 (1983) 217 [inSPIRE].

[13] S.G. Gorishnii and S.A. Larin, Coefficient functions of asymptotic operator expansions in minimal subtraction scheme, Nucl. Phys. B 283 (1987) 452 [inSPIRE].

[14] J.A.M. Vermaseren, Axodraw, Comput. Phys. Commun. 83 (1994) 45 [inSPIRE].

[15] O.V. Tarasov, A.A. Vladimirov and A.Yu. Zharkov, The Gell-Mann-Low function of QCD in the three loop approximation, Phys. Lett. B 93 (1980) 429 [INSPIRE].

[16] S.A. Larin and J.A.M. Vermaseren, The three loop QCD $\beta$-function and anomalous dimensions, Phys. Lett. B 303 (1993) 334 [hep-ph/9302208] [INSPIRE].

[17] K.G. Chetyrkin and A. Maier, Massless correlators of vector, scalar and tensor currents in position space at orders $\alpha_{s}^{3}$ and $\alpha_{s}^{4}$ : Explicit analytical results, Nucl. Phys. B 844 (2011) 266 [arXiv: 1010.1145] [INSPIRE].

[18] A.L. Kataev, N.V. Krasnikov and A.A. Pivovarov, Two loop calculations for the propagators of gluonic currents, Nucl. Phys. B 198 (1982) 508 [Erratum ibid. B 490 (1997) 505] [hep-ph/9612326] [INSPIRE].

[19] K.G. Chetyrkin, B.A. Kniehl and M. Steinhauser, Hadronic Higgs decay to order $\alpha_{S}^{4}$, Phys. Rev. Lett. 79 (1997) 353 [hep-ph/9705240] [INSPIRE]. 